\title{
A novel treatment of knee degenerative disorders all-in-one intra-articular injection of platelet-rich plasma combined with hyaluronic acid
} Purpose: Therapeutic evaluation of all-in-one intra-articular (IA) injections of autologous platelet rich
plasma (PRP) combined with hyaluronic acid (HA) for knee degenerative disorders.

Material and methods: The protocol consisted in one or three IA injections, generally ultrasound guided, of a combination of PRP and HA prepared with the innovative Cellular Matrix device (CMPRP-HA) on three cohorts of patients: A first cohort of 202 patients with meniscal lesions, comprising 93 patients with grade II or III stable meniscal tear, 59 patients after meniscal suture and 50 patients with meniscal cyst, a second cohort of 20 patients with Grade II or III osteoarthritis (OA) and a third cohort of 40 patients presenting post traumatic bone marrow edema (BME) comprising 20 patients with post-traumatic algodystrophy (PTA) and 20 patients with post-traumatic osteoarthritis (PTOA). The International Knee Documentation Committee (IKDC) subjective knee score and the Western Ontario and McMaster Universities Osteoarthritis Index (WOMAC) pain scale as well as magnetic resonance imaging (MRI) and ultrasounds were used to assess results.

Results: Patients with meniscal lesion were treated with one injection of CM-PRP-HA. The follow up evaluation was done after one year. Significant improvement in the IKDC score (79.6/100 vs. 42/100 before treatment) was observed for patients with meniscal tears. Patients with meniscal suture presented no failure, while the success rate for patients treated for meniscal cysts was $70 \%$. Patients with degenerative OA received 3 IA injections of CM-PRP-HA at day 0 , at 2 months and at 6 months. Significant difference in the WOMAC pain scale was observed during the final evaluation at 9 months compared with value at day 0 ( 2.45 vs. 5.65). Patients presenting post traumatic bone marrow edema were treated with one IA injection of CM-PRP-HA and evaluated after one month. The pain score decreases from 8 to 4 for PTA and PTOA patients. This result was correlated with a reduction of bone marrow edema observed with MRI.

Conclusions: The Cellular Matrix device has been designed to prepare intra-articular injections of a combination of PRP and HA for symptomatic treatment of articular pain and mobility improvement, essentially for patient suffering from knee OA. In this study, other indications for this innovative treatment are also proposed. CM-PRP-HA appears to be a more efficient alternative to visco-supplementation with $\mathrm{HA}$ for the symptomatic treatment of knee articular disorders, including pain reduction and increase of knee functionality for patients suffering of osteoarthritis, post traumatic bone marrow edema, meniscal tears, healing of meniscal suture and size reduction of meniscal cysts. Further investigations will determine the optimal frequency of IA injections with CM-PRP-HA, and whether this innovative product would represent not only a conservative treatment for various knee articular disorders but also a preventive treatment for $\mathrm{OA}$, thus delaying the need for knee surgery.

Keywords: platelet-rich plasma $\bullet$ hyaluronic acid $\bullet$ degenerative osteoarthritis $\bullet$ bone marrow lesions • bone marrow edema $\bullet$ meniscal lesions $\cdot$ regenerative medicine

\section{Introduction}

Knee is the most common location for symptomatic degenerative $\mathrm{OA}$ and the risk of developing this pathology is almost $40 \%$ for men and $47 \%$ for women [1]. To treat this frequent and expensive pathology, recommendations of all Academic Societies relate to the use of nonpharmacological and pharmacological therapies [2-4]. Intra-articular injection of corticosteroids only claims to be effective in the acute phases of OA, by reducing pain and articular effusion, thus allowing to dry up the joint. However, the frequency of these injections is limited due to the side effects of corticoids.

The interest of visco-supplementation with HA IA injections in managing osteoarthritic knee pain is well known [5-7]. HA is a major component of healthy synovial fluid. It acts

\author{
Philippe Adam*1, Jean Luc \\ Renevier ${ }^{2}$ \& Jean- Francois \\ Marc $^{3}$ \\ 'Head, Medical Imaging Department, \\ Medipole Garonne Sports Clinic - 45 rue \\ de Gironis, 31100 Toulouse, France \\ ${ }^{2}$ Health Center du Lac, René Dugay Trouin \\ Street, 78200 Mantes-la-Jolie, France \\ ${ }^{3}$ Private Practice, AGCOSS, 2 Elisée Reclus \\ Street, 42300 Roanne, France \\ *Author for correspondence: \\ adamphhj@yahoo.fr
}


as a lubricant and shock absorbing agent and contributes to joint homeostasis through various biomechanical and biological mechanisms, including cell-receptor interactions [5]. The loss of endogenous HA appears to contribute to joint pain and stiffness. The place of the viscosupplementation among current therapeutics agents seems to be a third alternative after failure of antalgic drugs and failure or intolerance to NSAIDs. However, the effectiveness of HA for $\mathrm{OA}$ is mainly on reducing pain and doesn't last more than a few months [6].

To improve the efficiency of IA medical treatment of OA, Sampson et al. [8] were the first to study the effect of IA injection of PRP in 14 patients with primary and secondary knee OA. These patients received 3 PRP injections at a four-week interval in the affected knee. There were no adverse events reported. Moreover, this study demonstrated significant and almost linear improvements in knee injury and OA outcome scores, including pain and symptom relief. The majority of the patients expressed a favorable outcome at 12 months after treatment.

Filardo et al. [9] treated 91 patients presenting with a chronic knee degenerative condition equally with three IA PRP injections with a follow up at 6, 12 and 24 months. Significant improvement was seen the first year however, all the evaluated parameters were significantly lower at 24 months with respect to the 12-month evaluation. The IKDC objective evaluation fell from 67 to $59 \%$ of normal and nearly normal knees while the IKDC subjective score fell from 60 to 51 . The median duration of the clinical improvement was 9 months even though the results at 24 months remained significantly higher that the value before treatment. These findings confirmed that treatment with PRP injections can reduce pain and improve knee function and quality of life with a longer efficacy than HA.

Subsequently, Anitua et al. [10] showed that PRP stimulated the biological properties of HA, and Guler et al. [11] evoked a favorable action of IA PRP by comparing its use with that of HA in early-stage knee arthritis. The 2015 study of Gobbi et al. [12] also showed that IA PRP injections used for symptomatic early stages of knee OA induced significant reduction in pain and improved function after 12 months, which can be further improved at 18 months by annual repetition of the treatment. The same year, Marmotti et al. [13] reviewed the efficiency of IA injections with PRP for cartilage lesions and evoked the potential negative impact of the presence of high neutrophil content in leukocyte rich PRP.

A few clinical articles are concerned with the novel concept of combining PRP and HA injections for the treatment of degenerative disorders of the knee. This therapeutic association would take advantage of the synergistic anabolic actions of these two active substances introduced into the synovial environment. This is further supported by the fact that stimulation of collagen synthesis is correlated with growth factors and the regenerative effect of PRP on the chondrocyte [14-16].

Chen et al. [14] studied the effects of HA and $\mathrm{PRP}$ in an in vitro OA chondrocyte model and found that this association could rescue pro inflammatory cytokines-induced degeneration through chondrogenic signaling recovery. They strongly believed that PRP combined with HA could attenuate cartilage degeneration.

In the study by Sundman et al. [15], an ex vivo coculture system of OA cartilage and synoviocytes, both PRP and HA enhanced metabolism (decreasing catabolism) and diminished markers of inflammation and nociception (TNF $\alpha$ ). In synoviocytes, the use of PRP alone significantly decreased Matrix Metallo-Proteinase (MMP)-13 expression. MMP-13 is recognized as integral in cartilage matrix degradation during the development and continuation of OA.

These two studies were very helpful in understanding the mechanisms of this biological treatment which can produce a fundamental antalgic and anti-inflammatory effect by controlling the secretion of nociceptive and inflammatory mediators from cartilage and synoviocytes. Overall, an association of HA and PRP could act against the articular damage generated by trauma and degenerative $\mathrm{OA}$, and improve patient-reported pain and functional scores and, if successful, could delay or avoid surgery [16-19].

The Cellular Matrix device is the sole medical device that has been designed and certified for the preparation of PRP combined with HA in a manner conform to regulations and good practices. Two recent papers highlighted the effects of intra-articular injections of the Cellular Matrix PRP-HA combination for the treatment of patients suffering from knee OA [20,21]. Renevier et al. [21] reported the good results 
A novel treatment of knee degenerative disorders all-in-one intra- Research Article articular injection of platelet-rich plasma combined with hyaluronic

acid

of CM-PRP-HA in a pilot, multicenter French study with a long-term follow-up, after a series of three I-A injections scheduled at D0, D60 and D180, providing long-lasting benefits for half of the patients and avoiding surgery for almost $80 \%$ of them at four years. The Medipole Garonne Sports Clinic was one on the centers participating to this study.

The purpose of this work, performed in Medipole Garonne is to assess the current situation of the CM-PRP-HA protocol for the treatment not only of degenerative knee OA but also of other knee disorders and to determine practical indications for clinical use.

\section{Materials and methods}

\section{CM-PRP-HA protocol}

The Cellular Matrix (CM) A-CP HA Kit (Regen Lab SA, Le Mont sur Lausanne, Switzerland) is a Class III medical device (European classification). CM has been specifically approved for the single step preparation, from a small sample of patient's blood, of autologous PRP in presence of HA in a sterile closed-circuit system. The CM device is an evacuated tube in which the patient blood sample $(6 \mathrm{ml})$ is automatically collected. The blood-filled tube is centrifuged for 5 minutes at a relative centrifugal force of $1500 \mathrm{~g}$ (following the manufacturer's instructions). Thanks to the Regen Lab separating gel technology, the platelets and plasma are isolated from the other blood components and combined with the HA solution present in the device. The resulting CM-PRP-HA consists of around $3 \mathrm{ml}$ of autologous PRP, with a platelet concentration 1.5 to 1.6 times higher than the baseline value in venous blood and with a low contamination in red and white blood cells (neutrophil poor PRP), entrapped in a 3D network of HA. The device contains $2 \mathrm{ml}$ of natural, non-cross-linked HA at a concentration of $20 \mathrm{mg} / \mathrm{ml}$ (40 mg total). The HA is produced by bacterial fermentation, thus devoid of animal proteins. CM is approved for both orthopaedic and dermal applications in Europe, and clinical studies are ongoing in the US to obtain FDA pre-market approval.

CM-PRP-HA injection was always preceded by a medical consultation with analysis of clinical and imaging data, collection of patient consent and examination for infectious or haemorrhagic risks with temporary interruption of anticoagulants drugs if necessary. A blood count was also required. Systematic control MRI was done after the treatment. To reduce pain, premedication (antalgic drug and anaesthetic patch one hour before injection) and inhalation of analgesic gas (Entonox ${ }^{\oplus}$ MEOPA, Linde Healthcare, during injection) were systematically used.

The I-A injection (generally US guided) of the CM-PRP-HA combination was performed just a few minutes after its preparation in the same room, with all aseptic precautions during the entire procedure. The overall procedure is simple, easy and relatively short, typically lasting less than 20 minutes. NSAIDs were prohibited one month before and after IA injection to avoid hampering the PRP effect on the healing process.

Selected pathologies

Meniscal cohort

The meniscal cohort included patients with stable meniscal tear and patients after meniscal suture or with meniscal cyst. The first group of patients suffered from grade II and III meniscal degenerative tears with an unsatisfactory clinical response to standard medical treatment (NSAIDs or HA alone). MRI classification of Stoller et al. [22] was used before and after the treatment: Grade I lesion is described as nodular intrameniscal hypersignal, Grade II as linear intrameniscal hypersignal without articular surface extension, and Grade III as linear intra-meniscal hypersignal with extension towards at least one articular surface (e.g. meniscal tear, unhooking sign). The main clinical criterion for patient inclusion was a stable meniscal lesion into a stable knee. The IKDC subjective knee score ("well-being" scale between 0 and 100) [23] evaluated reliability, validity, and responsiveness to the CM-PRP-HA treatment.

From August 2012 to June 2013, 93 patients (aged between 23 and 84 years, mean age 49 , gender ratio: $24 \%$ female vs $75 \%$ male) suffering from Grade II or III ( $80 \%$ grade III) stable horizontal lesion (85\% medial meniscus, $15 \%$ lateral meniscus, RR or RW meniscal area) were treated with only one IA injection of CM-PRPHA. If effusion was present, an arthrocentesis was done before injecting the product into the joint. All injections were US-guided into the joint by sub-patellar way. The IKDC score for this group was evaluated at a final follow up in August 2015.

A second group of 59 patients (50 men, 9 women, mean age 25 years) benefited of one injection of CM-PRP-HA at one month after surgical meniscal suture for bucket handle lesion ( 40 cases) or unstable meniscal flap (19 cases), in 
an attempt to strengthen meniscal healing and to reduce failures of surgery with recurrence of an instable tear [24].

US guided mechanical treatment of meniscal cysts was also performed for 50 patients with drilling and emptying of the cyst followed by a single injection of CM-PRP-HA into the joint by patellar way. Our purpose was to avoid the surgical ablation of the cyst and meniscus by stimulating the healing of the meniscal tear and the communicating channel.

Knee degenerative OA cohort

From September 2013 to April 2014, 20 patients (13 males and 7 females, aged between 40 and 77 years, mean age 59 years, mean BMI $25,83)$ suffering from knee OA of Kellgren and Lawrence [25] grade II (10 patients) and III (10 patients) were enrolled in Medipole Garonne as part of the multicenter French study of Renevier et al. [21]. Other selection criteria were failure to respond to HA treatment in the previous 3 months, and not taking analgesics or NSAIDs or anti-OA medication in the previous 3 months.

Therapeutic injections with CM-PRP-HA were performed by sub-patellar access at Day 0 , Month 2 and Month 6 and evaluated at these three time-points using the WOMAC scale (Visual Analogue Scale (VAS) for pain, 0-10 $\mathrm{cm})$ [26] WOMAC evaluation was also done at a final follow-up at Month 9. If effusion was present, an arthrocentesis was performed before injecting the product into the joint.

MRI was performed before the first injection in order to characterize the pathology, and at the end at 9 months. Only one MRI feature was noted: namely the presence or absence of Bone Marrow Edema (BME) before and after the treatment. BME was found in bone under areas where cartilage was damaged and was correlated with Bone Marrow Lesions (BML). MR Fat Sat PD-weighted sequences were always performed in the same order to detect the hyper-signal assimilated to edema femoral and/or tibial BME.

\section{Bone marrow edema cohort}

Twenty patients (13 men and 7 women) presenting condylar $(\mathrm{N}=15)$ or tibial $(\mathrm{N}=5) \mathrm{BME}$ with PTA following surgery for cruciate ligament repair received one US-guided IA injection of CM-PRP-HA. Two criteria were noted at one month, namely VAS pain score and decrease or disappearance of BME via MRI.

Twenty patients with PTOA, 16 men and 4 women, presenting medial $(\mathrm{N}=15)$ or lateral $(\mathrm{N}=5)$ condylar BME, associated with an infracentimeter superficial osteochondral defect, received one US-guided IA injection. Two criteria were noted at one month, namely VAS pain score and decrease or disappearance of BME around traumatic osteochondritis via MRI.

\section{Results}

\section{Meniscal cohort}

For patients with grade II and III degenerative meniscal tears there was a significant improvement in the IKDC subjective score one year after the beginning of CM-PRP-HA treatment, with a mean score of 79.6 (range 50 to $100 / 100$ ) compared with 42 (range 0 to 60/100) before CM-PRP-HA. Improvement of meniscal tear (partial or total reduction of the tear with decrease of grade III towards grade II, reduction of peripheral cyst around the meniscal wall), and reduction of associated signs (synovial and collateral ligament hyper-signal, joint effusion) were always correlated with clinical improvement (Figure 1). It should be noted that 10 patients $(10 / 93)$ failed to respond to the treatment and required surgery (1 suture and 9 partial meniscectomy) due to a poor evaluation of meniscal stability before the beginning of the study. A follow-up study at 2 years in August 2015 revealed that $52 \%$ of subjects exhibited long-term improvement with no severe adverse events reported.

For meniscal sutures injected after surgery, we found no failure of meniscal suture at the one year follow up, which indicates that CMPRP-HA seems to enhance healing of meniscal sutures. For meniscal cyst, rate of success at one year was $70 \%$. Fifteen patients $(30 \%)$ had an unsatisfactory response to the treatment with pain and no reduction in size of the cyst, particularly when cyst emptying was not satisfactory at the beginning of the process.

\section{Knee degenerative OA cohort}

The results for the sub-cohort of patients from Medipole Garonne form the French multicenter study (21) were considered satisfactory with a reduction in pain at 9 months always correlated with a decrease or total resolution of BME observed by MRI (Figure 2). The hyper-signal assimilated to edema was always decreasing or disappearing. The variations for the WOMAC pain scale were also significant with a mean value of 5.65 at the beginning of the treatment, 3.8 at Month 2, 2.95 at Month 6 and 2.45 at Month 
A novel treatment of knee degenerative disorders all-in-one intra- Research Article articular injection of platelet-rich plasma combined with hyaluronic

acid

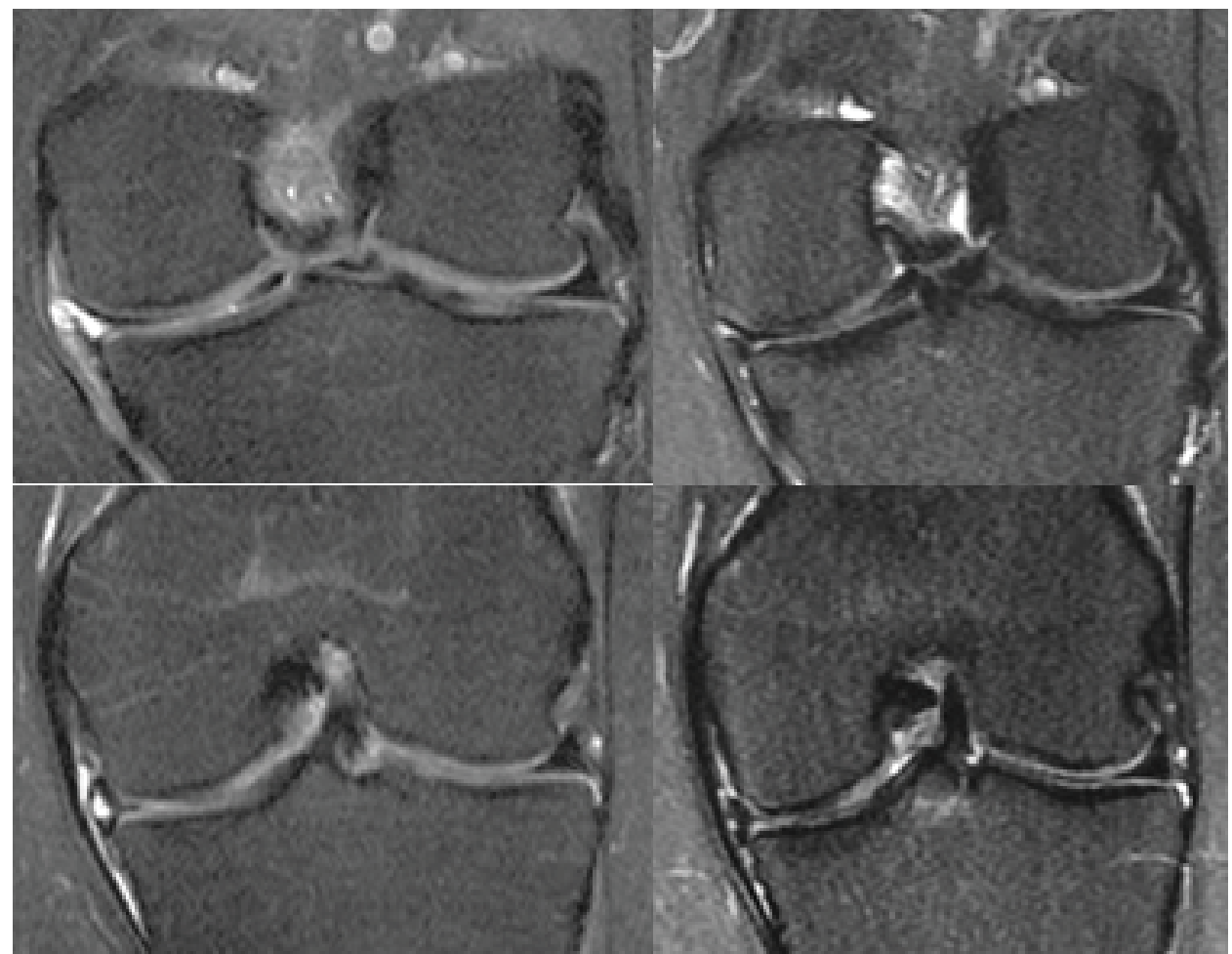

Figure 1. Grade III medial meniscus degenerative tear with peripheral cyst; Control MRI before (left side) and after CM-PRP-HA (five weeks) clearly demonstrate the decrease of meniscal wall edema, cyst and tear. Clinical improvement was correlated.
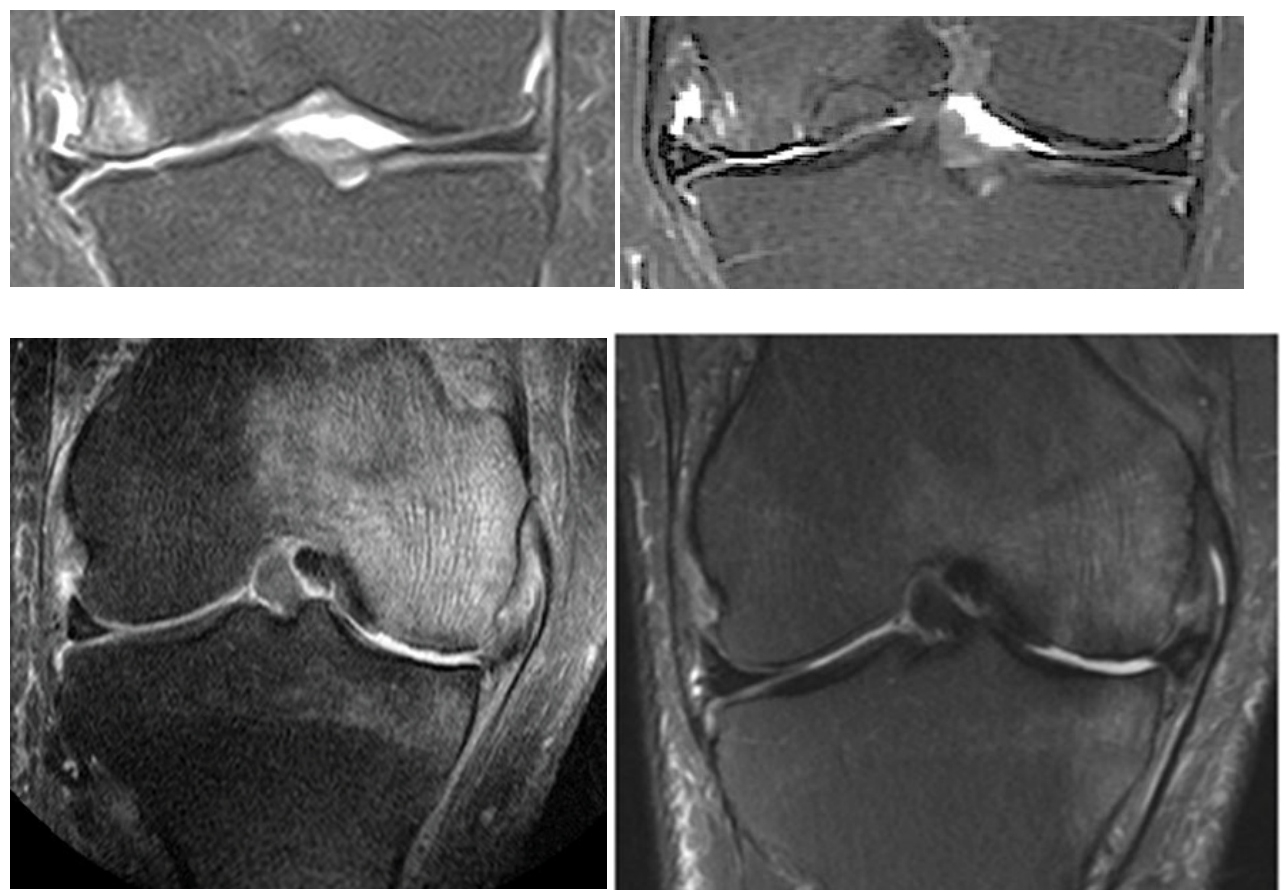

Figure 2. Two cases of Knee KL III OA; MRI was performed before (left side) and after CM-PRP-HA (right side). Condylar BME obviously decreased, and degenerative meniscal lesion was improved (meniscus arthritis) with reduced pain. 
9 (Figure 3). Only two adverse events were recorded. They were inflammatory reactions which lasted 5 and 7 days respectively, after the first injection. They were treated with ice applications and analgesics.

In November 2017, approximately 4 years after the start of the OA study in Medipole Garonne, we saw in consultation half of patients (the other half was lost to follow up). Most of them had a satisfactory functionality, and had not undergone prosthetic device surgery. These patients were keen to repeat the treatment. Thus, we can confirm the long term efficiency of intra-articular injection with CM-PRP-HA in comparison with a standard visco-supplementation. Results in the sub-cohort from Medipole Garonne are parallel to that of global multicenter study [21] in which $94.4 \%$ of the treated patients for OA of the knee were considered responders to treatment based on the OMERACT-OARSI criteria [27].

\section{Bone marrow edema cohort}

For the 20 patients with PTA, the decrease of pain (mean VAS score reduced from 8 to 4) was always correlated with a reduction or disappearance of BME via MRI (Figure 4). For the 20 patients with PTOA, the results were similar, with a reduction of the hyper-signal ring around osteochondritis (target sign) (Figure 5). Reduction of BME was highly correlated with a reduction in pain. CM-PRP-HA was effective at one month, and the results remained good for a sample of 5 patients seen again at 6 months.

Since this study, CM-PRP-HA has been used on a regular basis in our clinic. A total of 2328 IA injection procedures with CM-PRP-HA were performed from August 2012 to June 2018 (Figure 6). The largest age group (62\%) was between 41 and 60 years of age, with a relatively small group aged less than 21 years $(1 \%)$. The anatomical distribution of IA injections is provided in Figure 7, with the majority for the

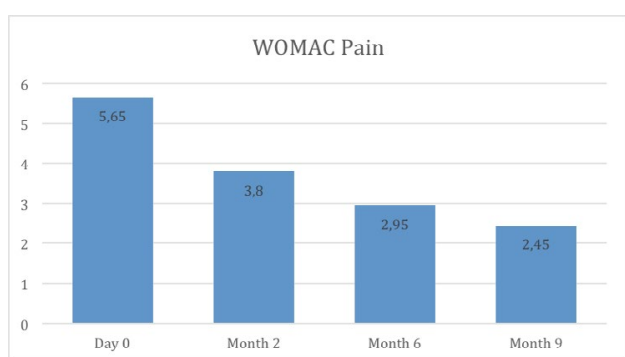

Figure 3. WOMAC Pain at Day 0, Month 2, Month 6 \& Month 9 after 3 sub-patellar CM-PRP-HA injections (sub-cohort of 20 patients from “Medipole Garonne").
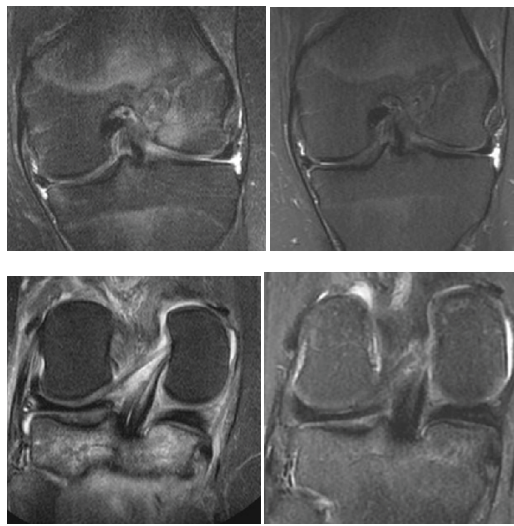

Figure 4. Knee PTA with BME. Lateral condylar edema along the tunnel of ligamentary plastic surgery (left top side). Tibial plate edema (left bottom side). Substantial improvement of the signal for cancellous bone after CM-PRP-HA at one month, correlated with decreased pain (right side).
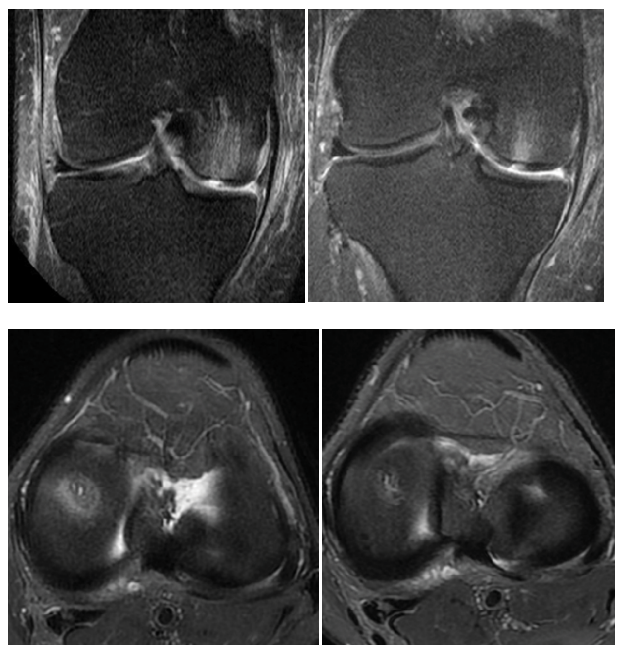

Figure 5. Knee PTOA with BME of medial femoral condyle and superficial cartilage defect (osteochondritis); Frontal plane (top) and axial plane (bottom) with a target sign. Edema highly decreased after CM-PRP-HA at one month.

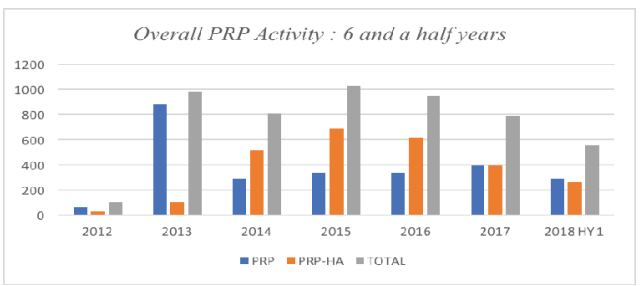

Figure 6. Global PRP therapy with Regen Lab devices; 2328 sessions of CM-PRP-HA from 2012/8 till 2018/6.

knee (84\%). The gender ratio was 36\% female vs $64 \%$ male.

\section{Discussion}

The literature supports the use of PRP IA 
A novel treatment of knee degenerative disorders all-in-one intra- Research Article articular injection of platelet-rich plasma combined with hyaluronic

acid

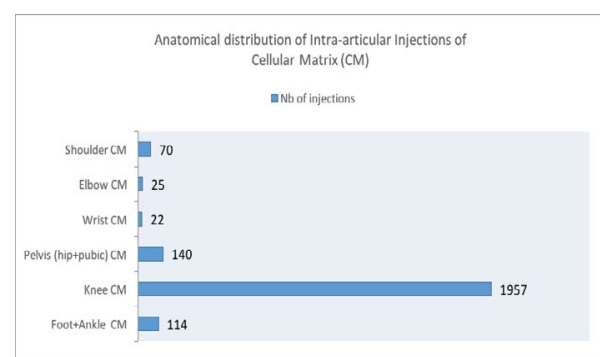

Figure 7. Anatomical distribution of CM-PRP-HA ( $84 \%$ for the knee, $6 \%$ for the hip, $4,9 \%$ for ankle and foot).

injections for the treatment of knee disorders [8-13]. In a recent review about the efficiency of I-A injection of PRP, Marmotti et al. [13] stated that PRP appears to have a potential for improving knee function and quality of life of patients suffering from chondropathy or initial $\mathrm{OA}$, by reducing the inflammation and, in a lesser extent, the degenerative articular processes. Nevertheless, better results are seen in younger male patients, with low BMI and lower degree of cartilage degeneration with protocols implying a repeated course of injections.

Medipole Garonne is a Sports Clinic where meniscal injuries are frequently seen. Our aim is to conserve the meniscus and avoid early primary degenerative $\mathrm{OA}$ and other pathologies caused by repeated traumas such as PTOA and meniscus arthritis. For these reasons, and with the aim of bringing a more effective and sustainable service to our patients, we propose to complement the therapeutic arsenal of degenerative disorders of the knee by the use of IA injection of CMPRP-HA. This proposal, particularly for young sportsmen, is in compliance with the statements of Marmotti et al. [13].

The question of whether CMPRP-HA was more effective than classical visco-supplementation can also be addressed from this study. According to the type of HA (viscosity), either a single or three IA injections separated by an interval of one week is recommended. In the study of Trigkilidas and Anand [6], the HA IA injections had a modest effect on early to moderate knee OA with the effect peaking at around 6-8 weeks following administration, with a doubtful effect at 6 months. In our clinical experience, cases of failure are also numerous for the treatment of knee OA with classical visco-supplementation. A new treatment option such as CM-PRP-HA, is thus necessary before opting for surgical solution like of tibial osteotomy or replacement with prosthetic device.
The need for treatment for BME and BML was first addressed by Davies-Tuck et al. [28], who stated that resolution of BML was associated with reduced progression of cartilage pathology. The presence of BML is associated with progressive cartilage loss and pain, thus $\mathrm{BME}$ is an important bio-marker however observable only by iterative MRI examination. Secondary MRI signs included the decrease of joint effusion and of soft para-articular tissues swelling. Consequently, the pattern of BME is an important consideration in degenerative followup that can only been observed with help of MRI, as X-ray cannot show cartilage and ignores the edema. The correlation of a reduction of pain and BME noted in the current study after CMPRP-HA treatment seems thus to be promising.

The best frequency for administering CM-PRPHA to maintain a good clinical result for pain beyond one, two, three or four years, and to avoid or postpone surgical intervention is still to be determined. In particular for sportsmen, should CM-PRP-HA injection be performed once each year, or a course of iterative IA injections with a two-month interval, as a preventive treatment for $\mathrm{OA}$ ?

\section{Conclusions}

The first key element for effective treatment of degenerative OA is the early detection and stabilization of fibro-cartilaginous tears since meniscal destruction is highly predictive for OA [8]. Secondly, the reduction of BME [28] favors healing, as BME is correlated with loss of cartilage. Therefore, early detection and early preventive treatment of OA might avoid the destructive evolution that could lead to the need for a prosthetic device.

Many favorable studies to the use of PRP, are already present in the recent literature: PRP vs placebo [29-30], PRP vs HA [31-34], a systematic review with comparison of the efficiency of PRP vs corticosteroid injections or visco-supplementation or placebo injections in knee osteoarthritis [35], and also the potential for PRP to activate sub-chondral progenitor cells [36]. We assume that neither PRP nor CMPRP-HA are placebos for the treatment of joint diseases.

The use of CM-PRP-HA has the potential to reduce pain more effectively than classical viscosupplementation, and to prevent, or at least to slow down, the progression of meniscal lesions and OA. Protection of fibro-cartilaginous 
structures is clearly coupled with protection of articular cartilage and future satisfactory state of the joint. Therefore, preventive treatment is extremely important to reduce pain, functional limitation and cost of public health. However, we cannot ignore the fact that being overweight, or having traumatic instability or distortions of the skeleton disadvantages the therapeutic benefits of any treatment.

Based on results obtained in this study we are able to propose the CM-PRP-HA protocol for the following pathologies:

- Grade II and III stable meniscal degenerative lesion in a stable knee with no meniscal extrusion, and with functional cruciate ligaments. Preventive treatment of meniscal lesions appears good for possibly avoiding meniscal arthritis.

- Kellgren and Lawrence Grade II and III Knee OA, and possibly Grade IV if surgery is refused by the patient.

- Post-Traumatic Algodystrophy of the knee.

- Post-Traumatic OA with BME and edematous lesions of superficial cartilage (osteochondritis).

In the future, a comparative analysis of CMPRP-HA vs HA visco-supplementation, as well as determining the most effective treatment regimen, will be probably useful.

\section{Disclosure of interest}

PA, JLR and JFM receive consulting fees from Regen Lab SA.

No funding was provided by Medipole Garonne Sports Clinic.

\section{Acknowledgement}

The authors would like to gratefully thank Marie Pierre Canal, Sébastien Desmaris and Laura Trevino Villa (Medipole Garonne Technologists) for their aid in performing the CM-PRP-HA treatments in this study.

\section{References}

1. Zhang Y, Jordan JM. Epidemiology of Osteoarthritis. Clin. Geriatr. Med. 26, 355-369 (2010).

2. Hochberg MC, Altman RD, April KT et al. American College of Rheumatology 2012 recommendations for the use of nonpharmacological and pharmacological therapies in osteoarthritis of the hand, hip and knee. Arthritis. Care. Res. 64(4), 465-474 (2012).

3. Chevalier. Reflections on the American College of Rheumatology recommendations for osteoarthritis from the French Section on Osteoarthritis: Comment on the article by Hochberg et al. Arthritis. Care. Res. 65(2), 324-328 (2013).
4. Jevsevar DS, Brown GA, Jones DL et al. The American Academy of Orthopaedic Surgeons evidence-based guideline on: treatment of osteoarthritis of the knee, 2nd edition. J. Bone. Joint. Surg. Am. 95(20), 1885-1886 (2013).

5. Huy AY, McCarty WJ, Masuda K et al. A systems biology approach to synovial joint lubrication in health, injury, and disease. Wiley. Interdiscip. Rev. Syst. Biol. Med. 4(1), 15-37 (2012).

6. Trigkilidas D, Anand A. The effectiveness of hyaluronic acid intra-articular injections in managing osteoarthritic knee pain. Ann. R. Coll. Surg. Engl. 95(8), 545-551 (2013).

7. Bellamy N, Campbell J, Robinson V et al. Viscosupplementation for the treatment of osteoarthritis of the knee. Cochrane. Database. Syst. Rev. 19(2), CD005321 (2006).

8. Sampson S, Reed M, Silvers $\mathrm{H}$ et al. Injection of plateletrich plasma in patients with primary and secondary knee osteoarthritis: a pilot study. Am. J. Phys. Med. Rehabil. 89(12), 961-994 (2010).

9. Filardo G, Kon E, Buda R et al. Platelet-rich plasma intra-articular injections for the treatment of degenerative cartilage lesions and osteoarthritis. Knee. Surg. Sports. Traumatol. Arthrosc. 19(4), 528-535 (2011).

10. Anitua E, Sanchez M, De la Fuente $M$ et al. Plasma rich in growth factors (PRGF-Endoret) stimulate tendon and synovial fibroblasts migration and improve the biological properties of hyaluronic acid. Knee. Surg. Sports. Traumatol. Arthrosc. 20, 1657-1665 (2012).

11. Guler O, Mutlu S, Isyar M et al. Comparison of shortterm results of intra-articular platelet-rich plasma and hyaluronic acid treatments in early-stage gonarthrosis patients. Eur. J. Orthop. Surg. Traumatol. 25, 509-513 (2015).

12. Gobbi A, Lad D, Karnatzikos G et al. The effects of repeated intra-articular PRP injections on clinical outcomes of early osteoarthritis of the knee. Knee. Surg. Sports. Traumatol. Arthrosc. 23(8), 2170-2177 (2015).

13. Marmotti A, Rossi R, Castoldi F et al. Review article. PRP and articular cartilage: a clinical update. BioMed. Res. Int. (2015).

14. Chen WH, Lo WC, Hsu WC et al. Synergistic anabolic actions of hyaluronic plasma and platelet-rich plasma on cartilage regeneration in osteoarthritis therapy. Biomaterials. 35, 9599-9607 (2015).

15. Sundman EA, Cole BJ, Karas V et al. The antiinflammatory and matrix restorative mechanisms of platelet-rich plasma in Osteoarthritis. Am. J. Sports. Med. 42, 35-41 (2014).

16. Siclari A, Mascaro G, Gentili C et al. A cell-free scaffoldbased cartilage repair provides improved function hyalinelike repair at one year. Clin. Orthop. Relar. Res. 470, 910-919 (2012).

17. Smyth NA, Haleem AM, Murawski CD et al. The effect of platelet rich plasma and hyaluronic acid on autologous osteochondral transplantation: an in vivo rabbit model. ORS (Orthopaedic Research Society) Annual Meeting 2014, New Orleans, poster n¹271.

18. Andia I, Abate M. Knee osteoarthritis: Hyaluronic acid, platelet rich plasma or both in association. Expert. Opin. 
A novel treatment of knee degenerative disorders all-in-one intra- Research Article articular injection of platelet-rich plasma combined with hyaluronic

acid

Biol. Ther. 14(5), 635-649 (2014)

19. Abate M, Andia I, Salini V. The conservative management of osteoarthritis- hyaluronic acid, platelet rich plasma or the combination? In: Prof. Qian Chen ed. Osteoarthritis Progress in Basic Research and Treatment, 2015.

20. Seleem NA, Elshereef E, Elhosary AA et al. Intraarticular injections of platelet-rich plasma combined with hyaluronic acid versus hyaluronic acid alone in treatment of Knee Osteoarthritis. EJPMR. 4(4), 608-615 (2017).

21. Renevier JL, Marc JF, Adam P et al. "Cellular Matrix TM PRP-HA": A new treatment option with platelet-rich plasma and hyaluronic acid for patients with osteoarthritis having had an unsatisfactory clinical response to hyaluronic acid alone: Results of a pilot multicenter French study with long-term follow-up. Int. J. Clin. Rheumatol. 13(4), 226-229 (2018).

22. Stoller DW, Martin C, Crues JV et al. Meniscal tears: pathologic correlation with MR Imaging. Radiol. 163(3), 731-35 (1987)

23. Crawford K, Briggs KK, Rodkey WG et al. Reliability, validity, and responsiveness of the IKDC score for meniscus injuries of the knee. Arthroscopy. 23(8), 839844 (2007).

24. $\mathrm{Xu} \mathrm{C,} \mathrm{Zhao} \mathrm{J.} \mathrm{A} \mathrm{meta-analysis} \mathrm{comparing} \mathrm{meniscal} \mathrm{repair}$ with meniscectomy in the treatment of meniscal tears: the more meniscus, the better outcome? Knee. Surg. Sports. Traumatol. Arthrosc. 23(1), 164-170 (2015).

25. Petersson IF, Boegård T, Saxne $\mathrm{T}$ et al. Radiographic osteoarthritis of the knee classified by the Ahlbäck and Kellgren \& Lawrence systems for the tibiofemoral joint in people aged 35-54 years with chronic knee pain. Ann. Rheum. Dis. 56 (8), 493-496 (1997).

26. Choquette D, Bellamy N, Raynauld JP. A FrenchCanadian version of the WOMAC osteoarthritis Index. Arthritis. Rheum. 37(9): S 226 (1994).

27. Pham T, Van Der Heijde D, Lassere $M$ et al. Outcomes variables for osteoarthritis clinical trials: The OMERACTOARSI set of responder criteria. J. Rheumatol. 30, 1648 1654 (2003).

28. Davies-Tuck ML, Wluka AE, Forbes A et al. The development of bone marrow lesions is associated with adverse effects on knee cartilage while resolution is correlated with improvement-a potential target for prevention of knee osteo-arthritis: A longitudinal study. Arthritis. Res. Ther. 12(1), R10 (2010).

29. Patel S, Dhillon MS, Aggarwal et al. Treatment with platelet-rich plasma is more effective than placebo for knee osteoarthritis: A prospective, double-blind, randomized trial. Am. J. Sports. Med. 41(2), 356-364 (2013).

30. Smith PA. Intra-articular autologous conditioned plasma injections provide safe and efficacious treatment for knee osteoarthritis: An FDA-sanctioned, randomized, doubleblind, placebo-controlled clinical trial. Am. J. Sports. Med. 44(4), 884-891 (2016).

31. Sanchez M, Azofra J, Usabiaga J et al. A randomized clinical trial evaluating plasma rich in growth factors (PRGF-Endoret) versus hyaluronic acid in the shortterm treatment of symptomatic knee osteoarthritis. Arthroscopy. 28(8), 1070-1078 (2012).

32. Filardo G, Kon E, Di Martino A et al. Platelet-rich plasma vs hyaluronic acid to treat knee degenerative pathology: Study design and preliminary results of a randomized controlled trial. BMC. Musculoskeletal. Disorders. 13, 229 (2012).

33. Cerza F, Carni S, Carcangiu A et al. Comparison between hyaluronic acid and platelet-rich plasma, intra-articular infiltration in the treatment of gonarthrosis. Am. J. Sports. Med. 40(12), 2822-2827 (2012).

34. Vaquerizo V, Plasencia MA, Arribas I et al. Comparison of intra-articular injections of plasma rich in growth factors (PRGF-Endoret) versus durolane hyaluronic acid in the treatment of patients with symptomatic osteoarthritis: A randomized contolled trial. Arthroscopy. 29(10), 16361643 (2013)

35. Meheux CJ, McCulloch PC, Lintner DM et al. Efficacy of intra-articular platelet-rich plasma injections in knee osteoarthritis: A systematic review. Arthroscopy. 32(3), 495-505 (2016)

36. Kreuz PC, Krüger JP, Metzlaff $S$ et al. Platelet-rich plasma preparation types show impact on chondrogenic differentiation, migration, and proliferation of human subchondral mesenchymal progenitor cells. Arthroscopy. 31(10), 1951-1961 (2015). 